\title{
Differentiating ZIP Codes in Syndromic Data; What Can They Tell Us?
}

\author{
Marc Paladini, Romona Lall and Stephen E. Schachterle* \\ New York City Department of Health and Mental Hygiene, Queens, NY, USA
}

\section{Objective}

To classify visits to NYC emergency departments (ED) into NYC residential, NYC PO Box or commercial building, commuters to NYC, and out-of-town visitors. To describe patterns in each group, to evaluate how they differ, and to consider how the differences can affect syndromic surveillance analyses and results.

\section{Introduction}

The NYC Department of Health and Mental Hygiene (DOHMH) ED syndromic surveillance system receives data from $95 \%$ of all ED visits in NYC totaling 4 million visits each year. The data include residential ZIP code as reported by the patient. ZIP code information has been used by the DOHMH to separate visits into NYC and non$\mathrm{NYC}$ for analysis; and, a closer examination of non-NYC visits may further inform disease surveillance.

\section{Methods}

Visits were initially differentiated into six home ZIP code types. NYC residential ZIP codes, PO Boxes and commercial buildings were identified with 2010 US Census and data from the SAS institute (SAS Institute Inc., Cary, NC, USA). Commuter visits to the EDs were classified as any ZIP codes from the NYC Core Based Statistical Area (CBSA; United States Office of Management and Budget). Out of town visits were identified using with the 2010 US Census. Unknown ZIP codes included all of those ZIP codes that were not identified by any of the previous methods, and missing ZIP codes were those that were blank. ZIP codes were verified with the United States Postal Service website (www.usps.com).

Once ZIP codes were categorized, spatial and temporal trends in total ED visits by home ZIP code type were analyzed.

\section{Results}

Of the approximately 4 million ED visits in NYC during 2011, the number of visits by commuters and out-of-town visitors were 125,236 $(3.1 \%)$ and $45,158(1.1 \%)$ respectively (Figure 1$)$. There were 4,676 $(0.1 \%)$ visits with a NYC PO Box or building ZIP codes and 48,077 $(1.2 \%)$ visits with a missing or non-interpretable ZIP codes. The majority of commuter and out-of-town ED visits occur at a smaller set of hospitals. Out-of town visitors mostly visited hospitals in Manhattan rather than hospitals in the outer boroughs. While the seasonal trends and day-of-week patterns for the NYC residents and the commuters appear to be fairly similar, this is not the case for out-of-town visitors. For example, total ED visit trends correlated well for NYC residents and commuters $(\mathrm{r}=0.77)$, but there was no correlation between NYC residents and out-of-town visitors $(r=-0.18)$ over time. The number of ED visits among out-of-town visitors was higher during summer months and the winter holiday season, and this trend may have reflected the larger number of visitors during these periods. Day- of-week patterns were similar for NYC residents and commuters with weekdays associated with larger numbers of visits compared to weekends. However, the opposite was found true for out-of-town visitors with larger number of visits occurring over the weekends compared to weekdays.

\section{Conclusions}

Considerable differences in temporal trends were found among out-of-town visitors, NYC residents, and commuters to NYC. Out-oftown visitors also tend to visit EDs located in Manhattan rather than in the outer boroughs. These results suggest that out-of-town visitors represent a unique population ED visitors. Analyzing NYC residents, commuters, and out-of-town visitors separately may provide additional information that could prove useful to daily syndromic surveillance activities.

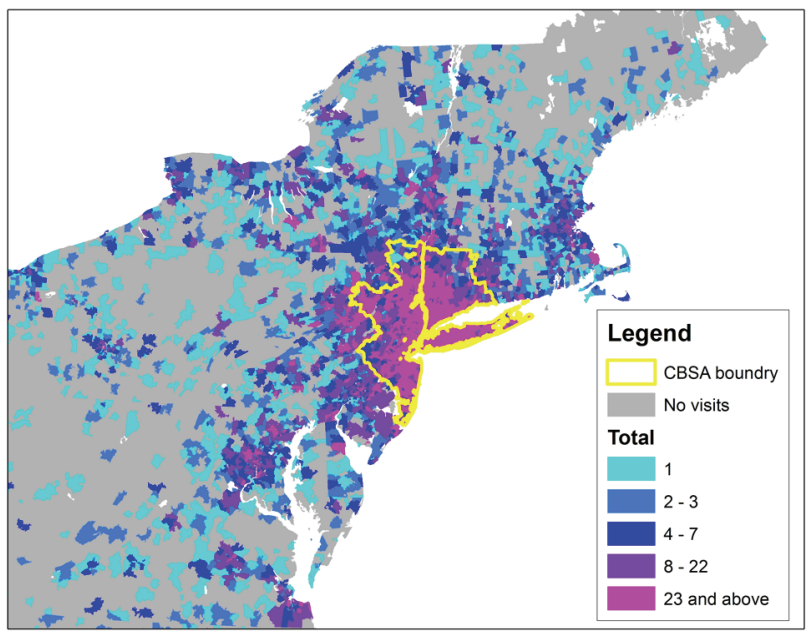

Figure 1: Total ED visits from the NYC Core Based Statistical Area (CBSA) and the surrounding region.

\section{Keywords}

GIS; New York; Spatial Analysis

\section{Acknowledgments}

This work was carried out in conjunction with a grant from the Alfred P. Sloan Foundation (\#2010-12-14). We thank the members of the New York City Department of Health and Mental Hygiene Syndromic Surveillance Unit.

\section{*Stephen E. Schachterle}

E-mail: steve.schachterle@gmail.com 